\title{
Performance of the ATLAS Jet Trigger
}

\author{
L. Lopes On behalf of the ATLAS Collaboration
}

\begin{abstract}
ATLAS is one of the general purpose detectors of the Large Hadron Collider (LHC) at CERN. The ATLAS trigger system, composed of three levels, is designed to cope with the LHC's demanding requirements. There have been a lot of recent changes in the ATLAS jet trigger. The standard strategy, based on Regions Of Interest, is not well-suited for multi-jet events since it leads to pathologies and efficiency losses. This philosophy has been changed for the jet trigger, introducing the possibility of unpacking the full calorimeter at the Event Filter and (even for a small subset of the events) at Level-2. We also moved to the use of calibrated energy scale at the trigger level, and to the application of noise cuts to reduce rate spikes. We will present the performance of the jet trigger in 2011, when most of these changes were operational
\end{abstract}

\section{Jet Trigger Motivation}

$\mathbf{T}$ HE Large Hadron Collider (LHC) currently provides proton-proton ( $\mathrm{pp}$ ) collisions at a bunch crossing rate of $20 \mathrm{MHz}$, having progressively increased the luminosity during 2011 and 2012, up to $3.65 \times 10^{33} \mathrm{~cm}^{-2} \mathrm{~s}^{-1}$. A Toroidal LHC Apparatus (ATLAS) [1] is one of LHC's general purpose detector with a comprehensive physics program.

The ATLAS Trigger and Data Acquisition (TDAQ) system (Figure 1p has to cope with stringent requirements of the LHC? on one hand being able to deal with high event rates, on the other hand selecting interesting physics events bringing down the event rate to around $400 \mathrm{~Hz}$, complying with data storage 4 capability.

Since it started operation in November 2009, the LHC has been working very well, delivering $5.6 \mathrm{fb}^{-1}$ of $7 \mathrm{TeV}$ pp collisions and since 2012 (until mid-April), $2.3 \mathrm{fb}^{-1}$ of $8^{5}$ $\mathrm{TeV}$ pp collisions.

Jets are the most common objects produced at the $\mathrm{LHC}^{5}$ and many important physics analysis topics such as $\mathrm{QCD}^{60}$ top quark physics, Higgs searches, searches for SUSY, exotic models, etc. strongly depend on the jet trigger. Therefores 2 excellent performance of the jet trigger is fundamental tøo achieve the physics goals of the ATLAS program.

\section{DESCRIPTION OF THE JET TRIGGER}

The jet trigger is part of the TDAQ, which is designed in three levels: Level 1 (L1), Level 2 (L2) and Event Filter (EF). The L2 and EF are together referred to as the High Level Trigger (HLT). The trigger system allows high flexibility for the trigger configuration to adapt to different running, conditions and physics requirements.

The Level 1 is hardware based, made with custom designed electronics, FPGA and ASIC [2], and has access to coarses granularity information from the Calorimeter.

The L1 Jet trigger reconstructs jets with a sliding window6 algorithm applied to calorimeter towers of $0.2 \times 0.2$ in pseudorapidity and azimuthal angle $(\eta \times \phi)$. The algorithm

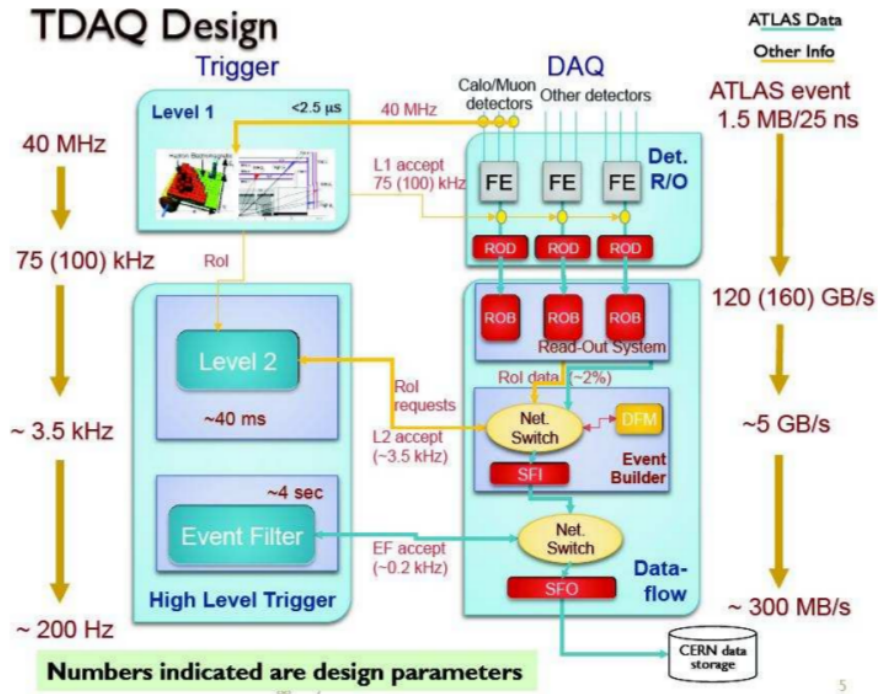

Fig. 1. ATLAS TDAQ architecture.

finds local transverse energy $\left(E_{T}\right)$ maxima, defining a Region of Interest (RoI) if $E_{T}$ is above a designated threshold. For the events passing L1 trigger, their RoIs are fed to L2.

L1 must provide a fast decision, it is designed to reduce the event rate from $40 \mathrm{MHz}$ to $75 \mathrm{kHz}$ within $2,5 \mu$ s of latency.

The Level 2 is software based. In the original design, jets are reconstructed using a simplified cone algorithm with radius $R=\sqrt{\Delta \eta^{2}+\Delta \phi^{2}}=0.4$ [3]. The algorithm accesses information from the calorimeter using the full granularity, around the seed provided by L1 RoI. L2 reduces the event rate from 75 to $3 \mathrm{kHz}$, within $40 \mathrm{~ms}$.

The EF uses sophisticated offline-like algorithms. It has access to the entire event data; i.e. reading out the whole calorimeter for events passing L2, and also benefits from full calibration of the detector. Topological clusters (three dimensional calorimeter clusters grouping offline-like cells), are used as input to the jet reconstruction performed using an anti-kT algorithm [4] with $\mathrm{R}=0.4$ and $\mathrm{R}=1.0$. The $\mathrm{EF}$ reduces the event rate from $3 \mathrm{kHz}$ to around $400 \mathrm{~Hz}$ for data storage, within about $4 \mathrm{~s}$ processing time.

A trigger chain is a combination of L1, L2 and EF triggers selections. The ATLAS detector selects events with several different jet topologies: events with at least one jet, multijets events or combinations of jets and other selection criteria such as missing transverse energy. Some of the jet trigger chains used during the 2011 proton-proton data-taking runs are summarized in Table 【 
TABLE I

EXAMPLES OF TRIGGER CHAINS RUNNING UNPRESCALED

\begin{tabular}{|l|c|c|c|}
\hline Trigger chains & $\begin{array}{c}\text { L1 } \\
(\mathrm{GeV})\end{array}$ & $\begin{array}{c}\mathrm{L} 2 \\
(\mathrm{GeV})\end{array}$ & $\begin{array}{c}\mathrm{EF} \\
(\mathrm{GeV})\end{array}$ \\
\hline \multicolumn{4}{|c|}{ Inclusive single-jet chains } \\
\hline \hline central jet & 75 & 95 & 240 \\
1 forward jet & 75 & 95 & 100 \\
\hline \hline \multicolumn{3}{|c|}{ Inclusive multi-jet chains } \\
\hline 3 central jets & $3 \times 50$ & $3 \times 70$ & $3 \times 75$ \\
5 central jets & $5 \times 10$ & $5 \times 25$ & $5 \times 30$ \\
\hline \hline \multicolumn{4}{|c|}{ Topological and combination chains } \\
\hline 1 central fat jet & 75 & 95 & 240 \\
anti-k $R=1.0$ & $2 \times$ & $2 \times$ & $2 \times$ \\
2 forward jets & $2 \times$ & 50 & 55 \\
with $\Delta \eta 5$ & 30 & 70 & 75 \\
1 central jet & 50 & +20 & +45 \\
$+E_{T}^{\text {miss }}$ & +20 & +20 \\
1 central jet & 75 & 95 & 100 \\
with $H_{T}>350$ & \multicolumn{2}{|c|}{} \\
\hline
\end{tabular}

\section{A. L2 Full Scan Jet Trigger implementation}

During 2011 the L2 jet trigger was redesigned introducing the L2 Full Scan (L2FS) also known as L1.5. It provides a new functionality for the $\mathrm{L} 2$ jet finding, reducing inefficiencies associated with the L1 sliding window algorithm in multi-jet events.

The readout time of all calorimeter cells is considerably high [5] and the time spent running any jet algorithm using cells as constituents is too large for the $\mathrm{L} 2$ processing time. Instead, in the new implementation, the L1 trigger towers are read directly from the L1 calorimeter readout system, enabling access to the entire event. This considerably reduces the granularity of the calorimeter information. In addition, the FastJet [6] algorithm was introduced at L2, configured to run anti-kT algorithm, the default jet algorithm in ATLAS. accessing L1 trigger towers, down to a minimum granularity of $0.1 \times 0.1$.

FastJet is a package with jet algorithms that can be used easily on many different jet constituents to run different algorithms in ATLAS.

In Figure 2 we present the current jet trigger architecture, including the new L2FS implementation. Results and the evaluation of the L2 Full Scan are further described in section IV

\section{Performance}

During 2010, the LHC established the center of mass energty for pp collisions at $7 \mathrm{TeV}$ and progressively increased the luminosity. High luminosity increases the event pile-up in thet detector, with typical values between 6 and 11 interactions pert bunch crossing in 2011, and 22 up to 40 in 2012.

The jet identification in the trigger is affected by pile-upe, due to the overlap of energy depositions in the calorimete causing an increase in the reconstructed $E_{T}$ of the jet. ${ }_{122}$

The jet trigger response for different pile-up scenarios wass evaluated performing efficiency and resolution studies. For4 $\mathrm{L} 1$, the efficiency was calculated as the fraction of offlines reconstructed jets that match a $\mathrm{L} 1$ trigger jet within 0.4 in $\Delta \mathrm{R}_{6}$ and that passes the corresponding trigger threshold. For $\mathrm{L}_{2,7}$

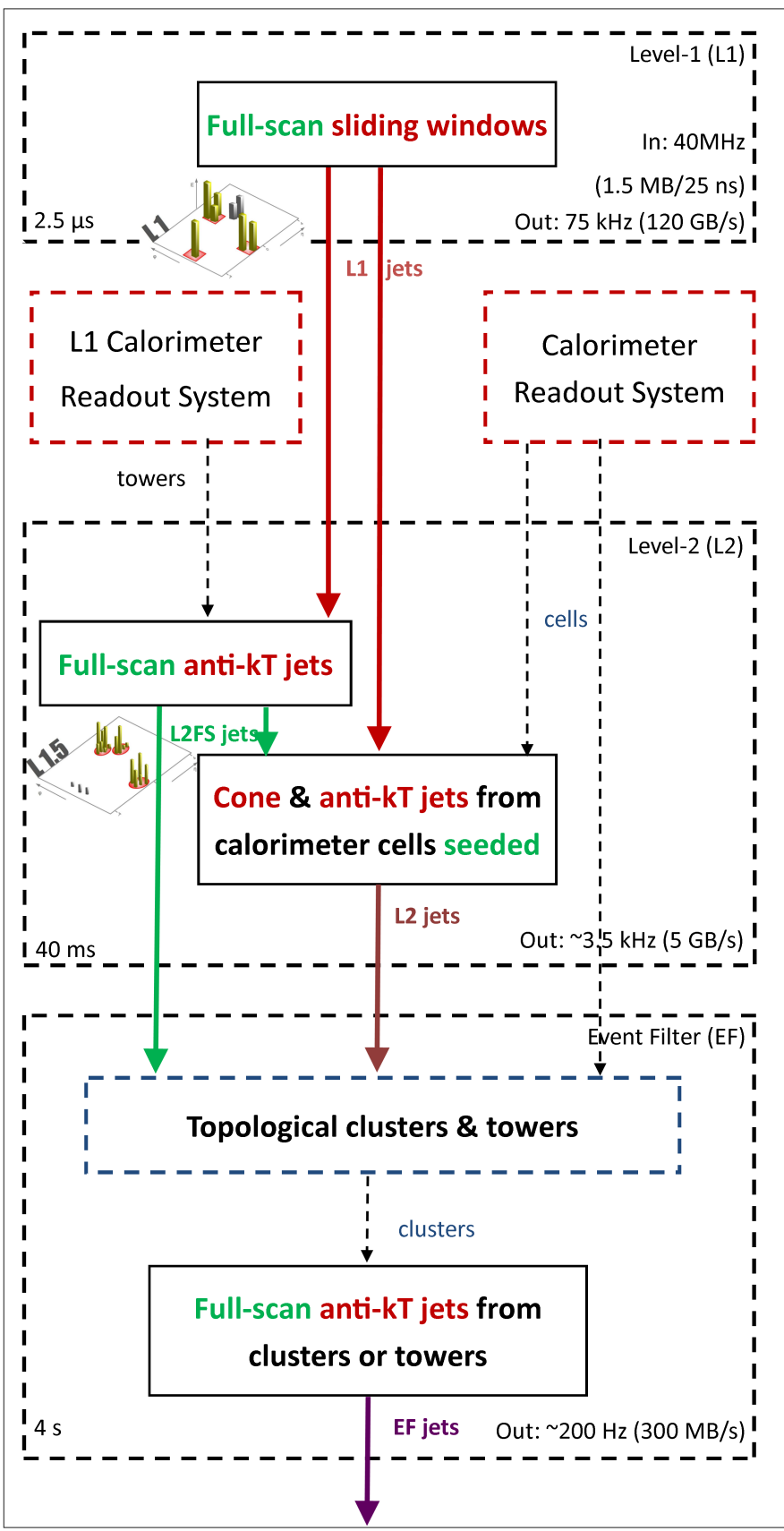

Fig. 2. Architecture of the ATLAS Jet Trigger with new L1.5.

again the efficiency was calculated as the fraction of offline jets that match a L2 jet, imposing in addition that the L2 jet matches a $\mathrm{L} 1$ jet. The $\mathrm{L} 2$ and $\mathrm{L} 1$ jets pass their corresponding trigger thresholds.

Since May 2011 onwards, a noise suppression tool, that considers electronics noise and pile-up noise, was introduced at L2 and EF. In figure 3 and 4, efficiencies for two different chains, with and without noise suppression are shown. For both chains, the sharpness of the turn-on curve was considerably improved with the introduction of the noise suppression, for the lower threshold trigger chain, the offline jet energy at which the L2 trigger efficiency reaches $99 \%$ was decreased by about $5 \mathrm{GeV}$. For the chains with the higher energy threshold 


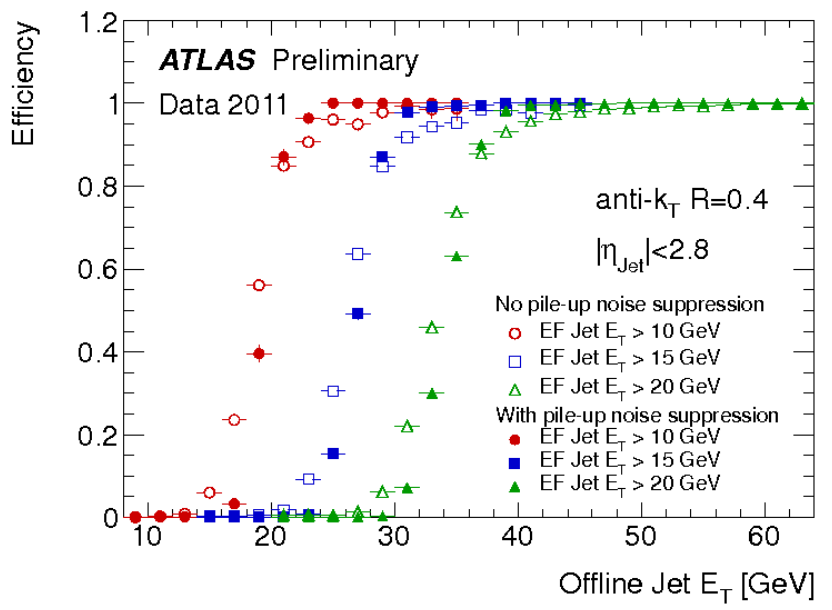

Fig. 3. The efficiency for anti-kt jets with $R=0.4$ to satisfy the Event Filter (EF) inclusive jet trigger for three choices of threshold. The EF-jet conditions were applied to random-triggered events. The efficiency is plotted as a function of the offline calibrated jet ET for jets with central rapidities and in two different data-taking scenarios: before (empty markers) and after (full markers) pile-up noise suppression was applied to EF-jets. (Jet energies in the trigger are measured at the electromagnetic scale.) [7]

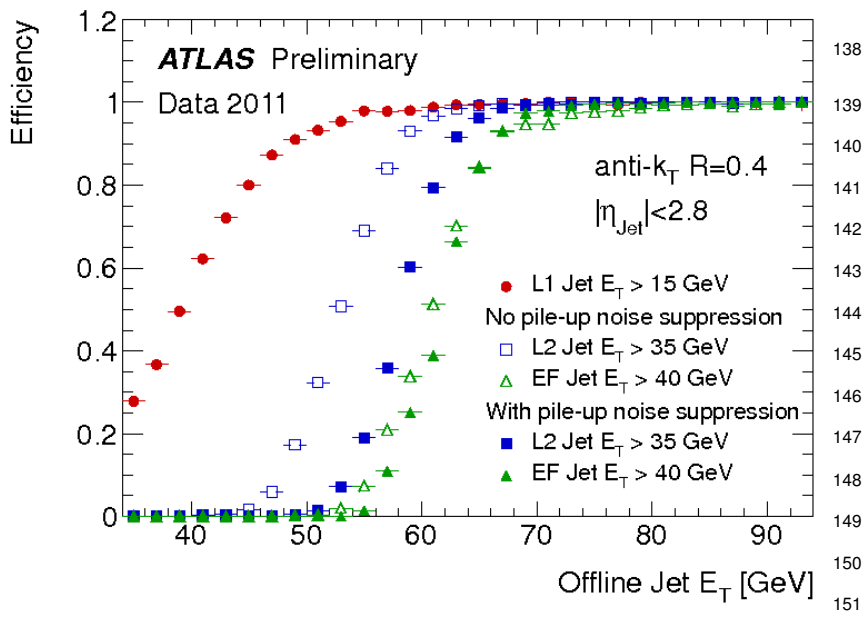

Fig. 4. The efficiency for anti-kt jets with $\mathrm{R}=0.4$ to satisfy Level 1 (L1), Level 2 (L2), and the Event Filter (EF) inclusive jet trigger for a single $\mathrm{L} 1->\mathrm{L} 2->\mathrm{EF}$ trigger chain. Different thresholds are applied at each leveq4 of the trigger to increase rejection of events while keeping acceptance f $9 \mathrm{~g}_{5}$ events with high probability of satisfying the overall jet trigger. The efficiency is plotted as a function of the offline calibrated jet ET for jets with central rapidities and in two different data-taking scenarios: before (empty marker597 and after (full markers) pile-up noise suppression was applied to both L258 and EF-jets. (Jet energies in the trigger are measured at the electromagnetic scale.) [7]

\section{9}

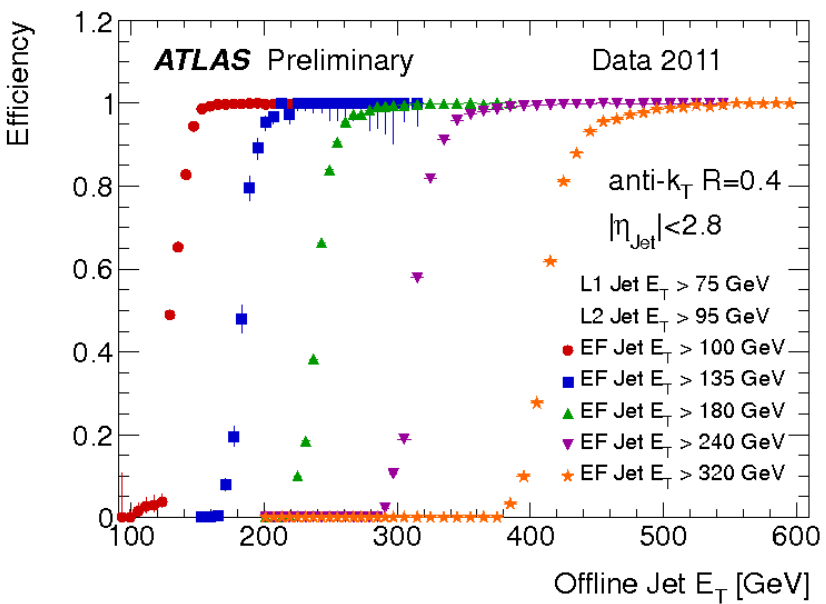

Fig. 5. The efficiency for anti-kt jets with $\mathrm{R}=0.4$ to satisfy the Event Filter (EF) inclusive jet trigger for five choices of threshold. The EF-jet conditions were applied to events selected with lower-ET jet triggers. The efficiency is plotted as a function of the offline calibrated jet ET for jets with central rapidities. These data correspond to the period when pile-up noise was suppressed during L2 and EF jet finding. (Jet energies in the trigger are measured at the electromagnetic scale.) [7]

\section{Level 2 Full Scan Performance}

For the heavy ion (HI) runs taken in 2011, the L2 Full Scan was run in the trigger. L2FS readout and jet finding timing studies were performed with $\mathrm{HI}$ data. Figure 6 and 7 show the L2FS jet finding and readout times for jets reconstructed using $0.1 \times 0.1$ and $0.2 \times 0.2 \mathrm{~L} 1$ trigger towers.

With lower granularity L2FS uses twice the average time to find the jets compared to the higher granularity case. Readout time is also higher for lower granularity although in both cases, L2FS processing time is kept within the designed $40 \mathrm{~ms}$ limit.

Position resolution and trigger efficiency of L2FS were studied for $p p$ collisions, re-running the HLT offline with L2FS triggering events. In figure 8 it is shown the efficiency response for L1, comparing L2FS with the RoI-based L1 for multi-jet events, as a function of the $6^{\text {th }}$ jet $p_{T}$. The full scan antikT reconstruction improves the efficiency for multi-jet event topologies by about $10 \%$.

Figure 9 shows the jet position resolution comparing the L1 jets, the L2FS jets with two different granularities, and L2 jets reconstructed by a cone algorithm. The L2FS position resolution is clearly improved as compared to L1.

\section{HEAVy IONS COLLISIONS}

In 2011, LHC delivered $\mathrm{Pb}-\mathrm{Pb}$ collisions at center of mass energy of $2.76 \mathrm{TeV}$. Heavy ion collisions are characterized by a parameter called centrality, which describes the central or tangential nature of impact between the colliding nuclei. More central collisions involve a larger number of colliding nucleons, resulting in a larger number of particles created and large underlying event activity. The flexibility of the EF

\footnotetext{
${ }^{1}$ Due to the non-compensation, the ATLAS calorimeters underestimate the energy deposited in hadronic showers. The electromagnetic scale measures correctly the energy deposited by electromagnetic showers. Additional calibrations have to be applied to hadrons and jets.
} 


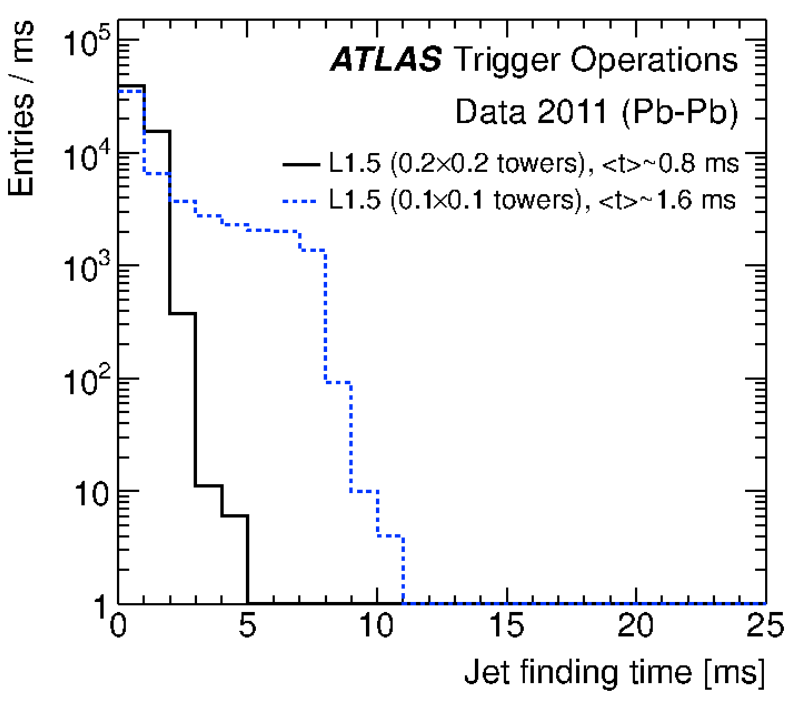

Fig. 6. The time taken to find jets using the anti-kT jet algorithm with a distance parameter $\mathrm{R}=0.4$. The black solid line shows the time taken for $0.2 \times 0.2$ (jet/Et miss) towers and the blue dashed line shows the time for $0.1 \times 0.1(\mathrm{gamma} / \mathrm{tau})$ towers. The timing was measured during $2011 \mathrm{~Pb}-\mathrm{Pb}$ collisions at $\sqrt{s_{N N}}=2.76 \mathrm{TeV}$. The nominal time limit at this stage of the trigger is about $40 \mathrm{~ms}$. [7]

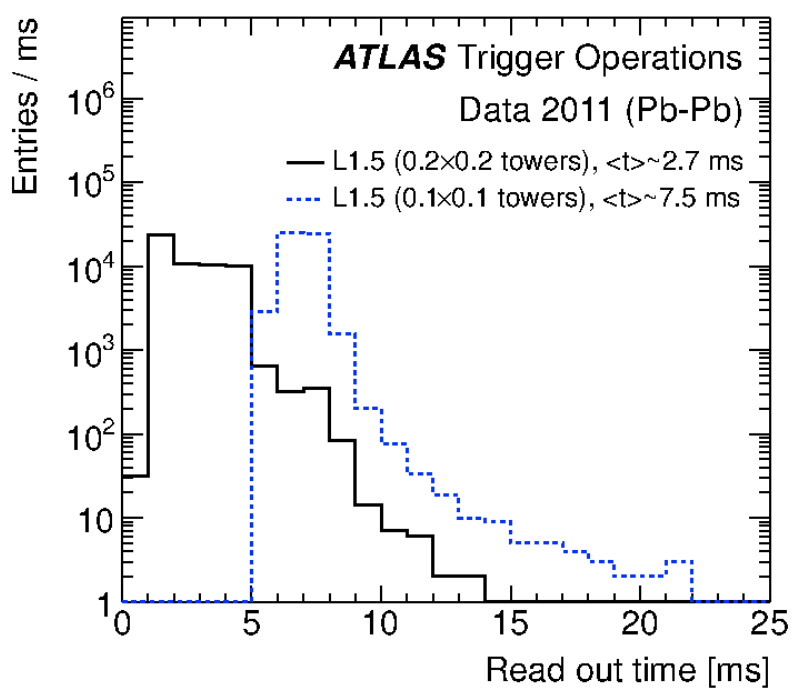

Fig. 7. The time taken to read out the tower information from the Level 1 calorimeter readout system. The black solid line shows the time taken to read out $0.2 \times 0.2$ (jet/Et miss) towers and the blue dashed line shows the time for $0.1 \times 0.1$ (gamma/tau) towers. The timing was measured during $2011 \mathrm{~Pb}-\mathrm{Pb}$ collisions at $\sqrt{s_{N N}}=2.76 \mathrm{TeV}$. The nominal time limit at this stage of the trigger is about $40 \mathrm{~ms}$. [7] configuration permitted to run offline dedicated underlying event subtraction algorithms, to correct the EF jet energies. ${ }_{177}$

Detailed studies of the jet trigger performance in $\mathrm{HI}$ runss were done. Figure 10 and 11 show the trigger efficiency and position resolution of different jet trigger selections for different centrality scenarios.

Although the trigger could be expected to be less efficiento for central collisions (lower values of centrality parameter), id is shown in figures 10 and 11 that neither the efficiency nor thez

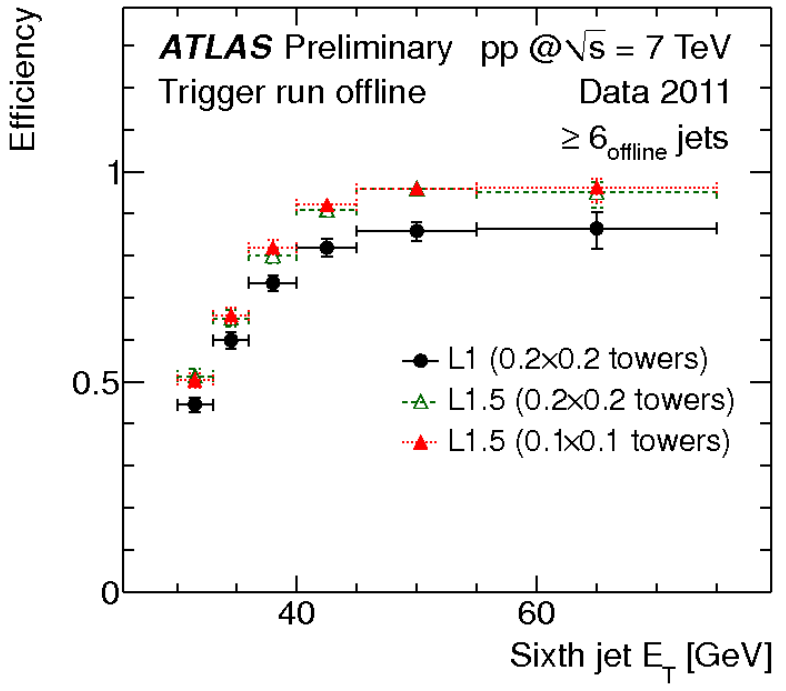

Fig. 8. The efficiency for L1 $(0.8 \times 0.8$ sliding window) and L1.5 (anti-kT $\mathrm{R}=0.4$ ) jets to satisfy a six jet trigger (trigger run offline) in events where at least six anti-kT $\mathrm{R}=0.4$ jets have been identified offline with $|\eta|<2.8$, $E_{T}>30 \mathrm{GeV}$ (these events were pre-selected using a four jet trigger). The efficiency is plotted as a function of the sixth offline jet ET. [7]

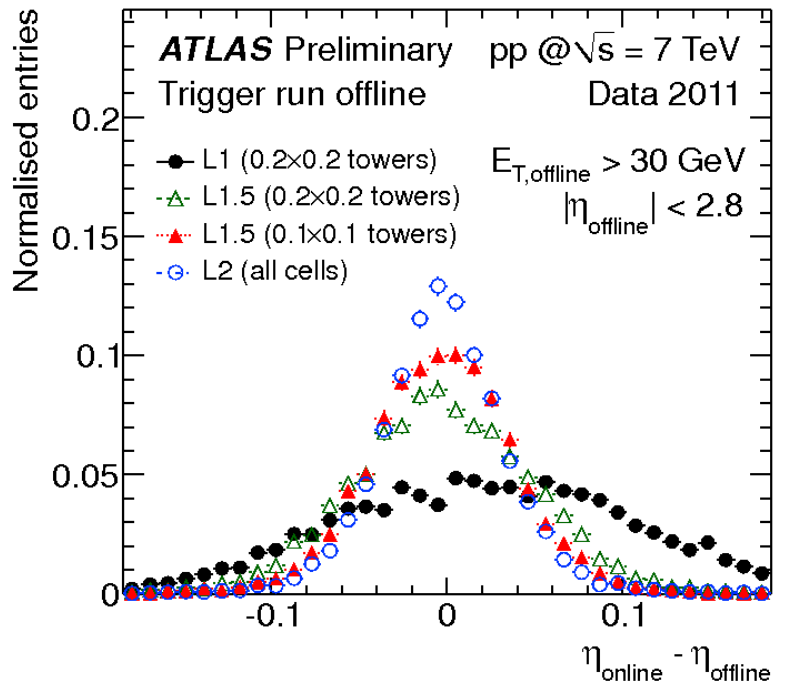

Fig. 9. The jet position resolution (in pseudorapidity) of the L1, L1.5 and L2 jet triggers in 2011 proton-proton collisions (trigger run offline). The jet finding algorithm for L1 is a $0.8 \times 0.8$ sliding window, for L1.5 it is anti-kT with $\mathrm{R}=0.4$ and for $\mathrm{L} 2$ a three-iteration cone $\mathrm{R}=0.4$ seeded by a $\mathrm{L} 1$ jet. The jet position resolution is evaluated with respect to offline anti-kT $\mathrm{R}=0.4$ jets. The offset toward high $\Delta \eta$ observed at L1 is an artifact of how L1 position is recorded. [7]

position resolution are affected by the centrality. In figure 10 jet energies in the trigger are measured at the electromagnetic scale $^{1}$.

\section{CONCLUSIONS}

Excellent performance of the ATLAS jet trigger system is essential for many physics analysis. The jet trigger is implemented in three levels that identify and select events with 


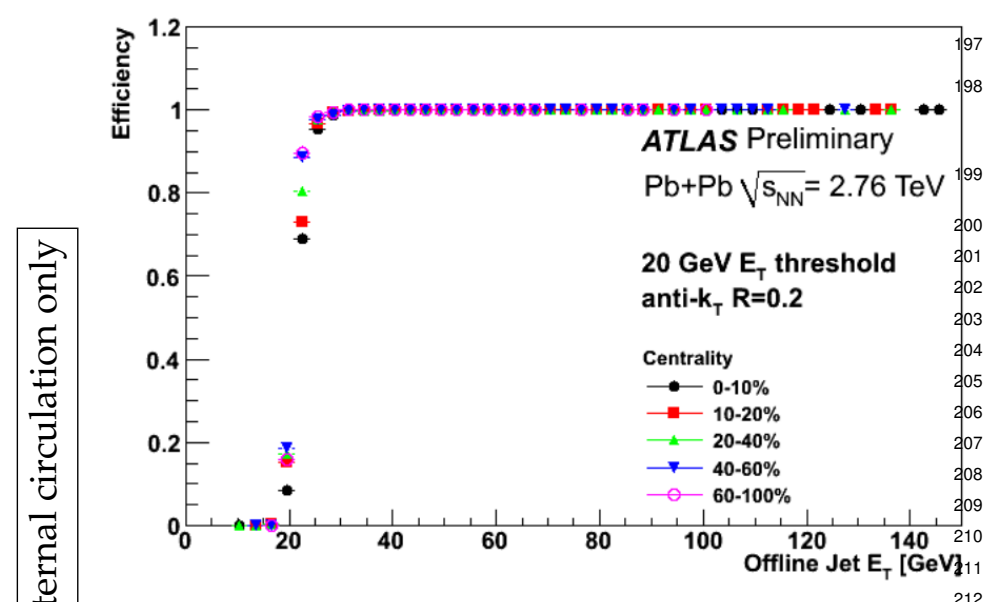

Fig. 10. Efficiency of the primary HLT jet trigger used for the 2011 heavy $i$ in $_{214}^{213}$ run. The efficiency was evaluated using the data from the $\mathrm{Pb}-\mathrm{Pb}$ collisions at $\sqrt{s_{N N}}=2.76 \mathrm{TeV}$ corresponding to an integrated luminosity of $2.4 \mu b^{-415}$ The HLT trigger algorithm is anti-kt $\mathrm{R}=0.2$ with a threshold of $E_{T}=20 \mathrm{Ge} \mathrm{V}_{217}^{116}$ The HLT trigger algorithm is seeded by events with total transverse energy greater than $10 \mathrm{GeV}$ identified by the Level 1 trigger. Efficiency is evaluated with respect to offline anti-kt $\mathrm{R}=0.2$ jets. Both the offline and HLT jets are at the electromagnetic scale. 7

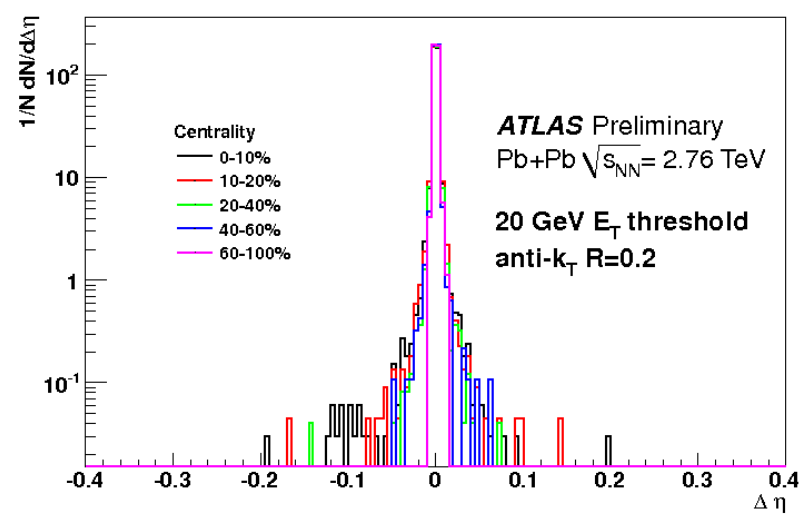

Fig. 11. Jet position resolution (in pseudorapidity) of the primary HLT jet trigger used for the 2011 heavy ion run. The jet position was evaluated using the data from the $\mathrm{Pb}-\mathrm{Pb}$ collisions at $\sqrt{s_{N N}}=2.76 \mathrm{TeV}$ corresponding to an integrated luminosity of $2.4 \mu b^{-1}$. The HLT trigger algorithm is anti-kt $\mathrm{R}=0.2$ with a threshold of $E_{T}=20 \mathrm{GeV}$. The HLT trigger algorithm is seeded by events with total transverse energy greater than $10 \mathrm{GeV}$ identified by the Level 1 trigger. The jet position resolution is evaluated with respect to offline anti-kt $\mathrm{R}=0.2$ jets. [7] high $p_{T}$ jets, both in the central and forward region of the detector. It is also flexible enough to select events based on different event topologies.

Recent changes to the jet trigger have greatly improved its performance. This was evaluated with data taken in 2011 and results show the ATLAS jet trigger is efficiently selecting events.

The noise suppression tool improves trigger acceptance by sharpening the efficiency turn-on curves.

The new L2 trigger implementation, L2FS, considerably improves L2 performance, especially for multi-jet topologies.

ATLAS has a very flexible trigger system that can be configured in different ways to adapt for the different physics requirements. This allowed to run dedicated underlying event subtraction for the heavy ion runs, improving considerably the trigger performance.

\section{REFERENCES}

[1] The ATLAS Collaboration, The ATLAS Experiment at the CERN Large Hadron Collider, Journal of Instrumentation 3 (2008) no. 08, S08003. http://stacks.iop.org/1748-0221/3/i=08/a=S08003.

[2] The ATLAS Collaboration, Performance of the ATLAS Trigger System in 2010. oai:cds.cern.ch:1388759, Eur. Phys. J. C 72 (Oct, 2011) 1849. 63 p. Comments: 50 pages, 64 figures.

[3] The ATLAS Collaboration, Expected performance of the ATLAS experiment: detector, trigger and physics, CERN-OPEN-2008-020, $<$ http://arxiv.org/abs/arXiv:0901.0512>arXiv:0901.0512.

[4] M. Cacciari, G. P. Salam, and G. Soyez, The anti-kt jet clustering algorithm, JHEP 04 (2008) 063, [arXiv:0802.1189].

[5] The ATLAS Collaboration, ATLAS experiment, Performance of the ATLAS Jet Trigger in the Early sqrt(s) $=7 \mathrm{TeV}$ Data, ATLAS-CONF2010-094, https://cdsweb.cern.ch/record/1299109.

[6] M.Cacciari et al, arXiv:1111.6097v1 http://arxiv.org/abs/1111.6097v1

[7] ATLAS Collaboration,

https://twiki.cern.ch/twiki/bin/view/AtlasPublic/JetTriggerPublicResults\# 2011_pp_Data_7_TeV 